\title{
Lumped Element Method - A Discrete Calculus Approach for Solving Elliptic and Parabolic PDEs
}

\author{
Zoltán Vizvári $^{1}$, Mihály Klincsik ${ }^{1}$, Zoltan Sári ${ }^{1}$, Péter Odry ${ }^{2}$ \\ ${ }^{1}$ University of Pécs, Szentágothai Research Centre \\ Ifjúság útja 20, H-7624 Pécs Hungary \\ vizvari.zoltan@mik.pte.hu,klincsik@mik.pte.hu, sari.zoltan@mik.pte.hu \\ ${ }^{2}$ University of Dunaújváros, Institute of Information Technology \\ Táncsics M. u. 1/A, H-2401 Dunaújváros, Hungary, podry@uniduna.hu
}

\begin{abstract}
In this report, we introduce a novel discrete calculus method for obtaining the numerical solution of parabolic and elliptic type partial differential equations. The discrete operators applied during the process of obtaining the numeric solution have the same advantageous properties as those of their continuous counterparts: orthogonality, conservation laws, and minimum-maximum principle. The results of our proposed solution method are interpreted in terms of the underlying physics and the material properties on the same graph. This can significantly simplify the solution of the discretised system that originates from the advantageous properties of discrete operators defined on weighted graphs. We demonstrate the applicability of the presented approach by using it to calculate the numeric solutions of an elliptic and a parabolic model problem and compare these results to the solutions of the same problems calculated using a well-known FEM solver.
\end{abstract}

Keywords: Discrete Calculus; Elliptic and Parabolic Problems; Differential Operators on Graphs; Lumped Elements

\section{Introduction}

Elliptic and parabolic partial differential equations (PDEs) are fundamental in the mathematics of physical laws. The practical impact of the solution of these kinds of equations is profound since many important physical phenomena used in various industrial applications are described by them. These problems are in disparate fields which include thermal energy transport, diffusion, electrostatics, electrodynamics etc. The general formulation of the aforementioned equations mentioned is as follows: 
$\frac{\partial u}{\partial t}+\nabla \cdot(-\kappa \nabla \mathrm{u})=s$

where $u=u(\boldsymbol{x}, t)$ is the unknown scalar field (potential), $s=s(\boldsymbol{x}, t)$ is the source term, $\kappa=\kappa(\boldsymbol{x}, t)$ is the isotropic transport coefficient, $\underline{x}=(x, y, z)$ and $\mathrm{t}$ are the space and time coordinates respectively, $\nabla u$ denotes the gradient of the scalar field $u$, and $\nabla \cdot \boldsymbol{v}$ is the divergence of the vector field $\boldsymbol{v}=-\kappa \nabla u$. The application of the weighted residual method (WRM) is a popular approach for solving these kinds of physical problems [1]. The application of WRM leads to several different well-known numerical methods such as the Finite Difference Method (FDM), Finite Volume Method (FVM), Finite Element Method (FEM), etc. All of these methods lead to an approximate solution, which satisfies the PDE and the boundary conditions as well [2]. Concerning the method of approximation, there are different approaches such as the variation principle, Galerkin-method, or the integral equations using the Green-function [2].

Another kind of distinction can be made based on the specific basis functions utilised for the various methods. Taking these into consideration, the following categories can be derived: discrete, semi-discrete and continuous [3]. In the discrete approach, the resulting approximate solution is defined only at discrete locations (points) in the investigated domain, which results, for example, in FDM. In this case, the solution satisfies the discretised equation in the interior nodes of the domain and the boundary conditions at all the boundary nodes as well. Thus, the solution is referred to as the strong form solution [2]. Semi-discrete methods use continuous basis functions that are defined on each of the discrete subdomains. The approximated solution consists of the linear combination of basis functions. The most popular method in this category is the FEM, which was first proposed by Ritz and Galerkin [2]. The finite element method determines the weak form solution to the governing PDE. This method was introduced first for problems in structural mechanics. Since then, researchers have exploited this technique for application to problems in other physical disciplines (for example fluid dynamics, heat transfer, etc.) [2]. In the continuous case (for example Analytic Element Method (AEM)), the approximated solution is defined on the entire domain (without discretisation). Currently, the AEM is the most applicable technique for groundwater simulations. In this case, AEM has some advantages over FDM/FEM which include precise estimation of the hydraulic head, generation of continuous flow solutions throughout the domain, and more accurate estimation of water budget, etc. [3].

Certainly, there are several other methods (for example Monte-Carlo Method [2], meshless, meshfree methods [2], Boundary Element Method (BEM) [2], etc.), but they are not relevant with regard to the purpose of this investigation.

During the numerical solution of PDEs several additional problems and difficulties can arise as follows: 
- Conservation laws: in some cases, neither semi-discrete nor continuous methods guarantee global conservation or local conservation $[4,5]$,

- Handling of complicated spatial geometries [4, 6, 7],

- Discontinuity or abrupt changes of material properties over the physical domain $[8,9]$,

- Complex-valued material properties, parameters, and field quantities, which can be effectively addressed by the proposed numerical method described in the following sections [10].

\subsection{Advantages of Discrete Calculus Methods}

The Discrete Calculus (DC) numerical approaches are able to effectively capture the physics of the underlying PDEs. Thus, compared to other methods (FEM, BEM, etc.), the DC Methods are more favoured by researchers in the field. The key feature of the application is the exact discretisation of the underlying physics and calculus [11] (before making any approximations) to exploit all the discrete differential operators (they mimic the mathematical properties of the continuous differential operators). [12-23] In general, the implementation of DC methods consists of two main steps: [4, 24]

1. Discretisation process: the continuous PDE system is substituted by its discrete counterpart,

2. Approximation process: solving the discrete system on the discretised domain.

The nature of a PDE (orthogonality, conservation, wave propagation, etc.) never depends on the details of the material. Thus, the DC approach always represents the physics of the PDE by transferring all numerical approximation errors to the material properties [11]. All approximations of material properties are based on the constitutive equations, which are strong physical statements. Errors in material properties do not affect the physical properties of a PDE system (for example local conservation of energy).

In general, a numerical solution method actually uses two meshes [24]. These include DC methods, FEM, FVM, and FDM as well as staggered mesh schemes. The solution is approximated on one mesh, and the equations are approximated on the other [24].

In this report, we propose a novel DC method to determine the solution of PDEs of the elliptic and parabolic type in particular. In order to carefully explain the novel approach, our report focuses only on the unsteady diffusion equation (1), which has the complexity to present the fundamental ideas of our DC approach. In the following, we establish the foundations of a novel discretisation method, which can be applied to the numerical solutions of various physical problems in the form (1). 


\section{The Proposed Method}

The novel method proposed by the authors incorporates the advantageous properties of the finite element and finite difference methods, thereby maintaining the simplicity of the discretisation approach of the finite difference method, while at the same time enabling arbitrary triangular discretisation of the domain.

The underlying principle of our method is to construct a topological operation, which forms a circular graph directly from a given physical domain (continuum). This way, not only the continuous domain is transformed into a graph, but the PDE of the problem will also be represented by discrete operators. The physical parameters become weights of the edges of the graph, and finally, the boundary conditions are represented by virtual edges connected to the boundary vertices of the graph (Fig. 1). By the application of discrete versions of the differential operators, the original problem can be directly discretised on a graph corresponding to an arbitrary triangular mesh. In this way, we do not attempt to obtain an approximate solution of the continuous problem, but instead, we solve the fully discretised version of the original problem directly and interpret the solution on the graph.

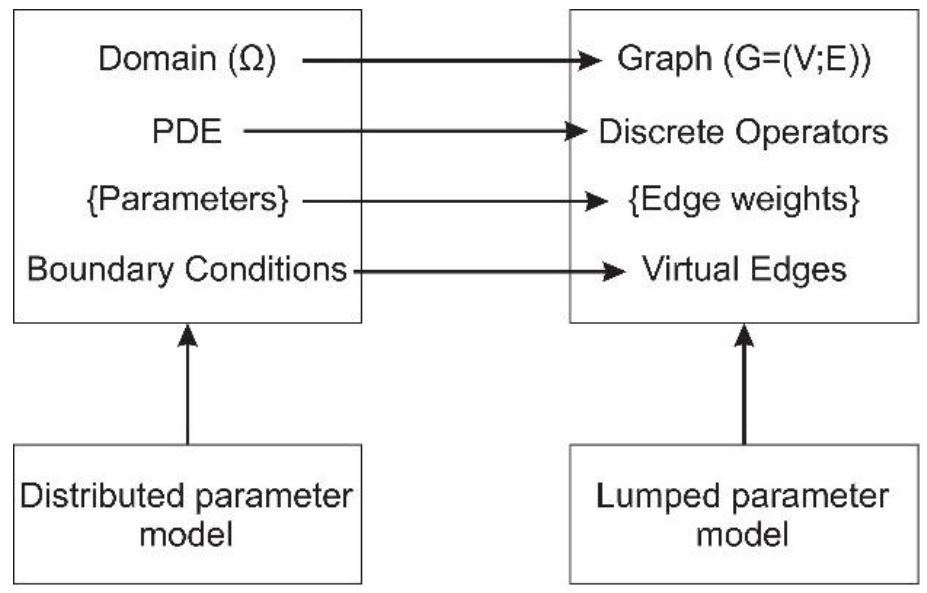

Figure 1

The concept of transformation 


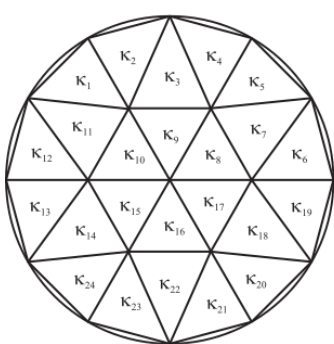

a

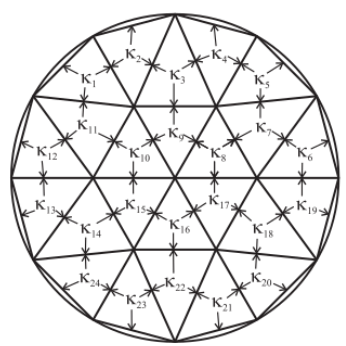

b

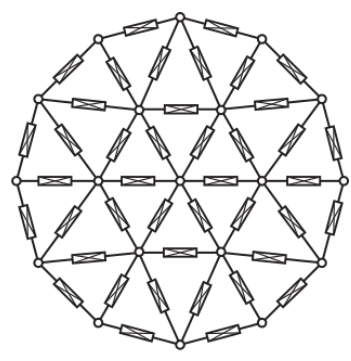

c

Figure 2

Transformation of the physical parameters $\kappa_{i}$ from the triangular domain to the edges of the triangles

$(a) \rightarrow(b)$, and representation by network $(c)$

As a result of this transformation, the material property values are lumped to the edges of the graph, and the original distributed parameter model becomes a discretised, lumped parameter model of the problem. As observed in Fig. 2, the continuous physical domain is subdivided into triangular domains, where the material properties $(\kappa)$ are assumed to be constant. The interface conditions are handled during the discretization of $\kappa$, where similarly to the nature of Fourierapproximation of functions having discontinouinity the $\kappa$ value on the interface will be equal to the average of the neighbouring domain values resulting in a kind of smoothing effect. Based on this approach, it is possible to interpret the physics (PDE) and the material properties on the same graph, which significantly simplifies the solution of the discretised system. The system of linear equations contains the properties of the Kirchhoff current law, so for interfaces, we assume continuity everywhere. This means that equal potential values are observed in the common nodes of triangles and the same fluxes in the case of their common sides.

\subsection{The Process of 'Lumping'}

In a three-dimensional case, Fig. 3(a) shows a tetrahedral element $T_{e}$ on which the set of lumped parameters is constructed from material property $\kappa$ on the domain.

The steps of the process are the follows:

1. Connect the centre of the circumsphere of the tetrahedral element with the middle-points of the edges. (Fig. 3(b))

2. The resulting sub-elements generally have irregular shapes, thus the crosssectional area $(A)$ perpendicular to the direction of flux is a function of the length along the direction of the flux. (Fig. 3(c))

3. The sub-elements can be handled as regular prisms with the same length and same volume $(V)$ as the original sub-element, while also having a constant cross-sectional area $(\bar{A})$, which is the average of $A$. (Fig. 3(d)) 
4. This way the one-dimensional model of conductivity can be applied. (Fig. $3(\mathrm{e}))$

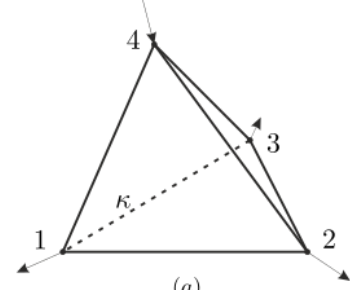

(a)

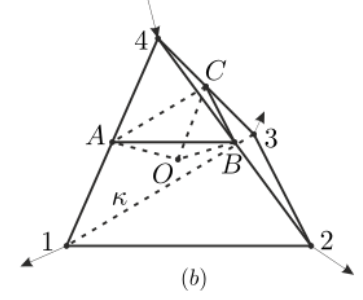

(b)

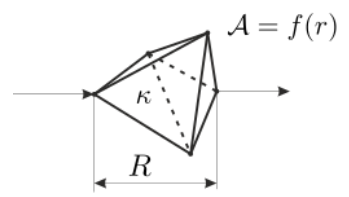

(c)

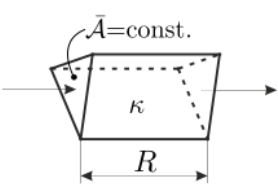

$(d)$

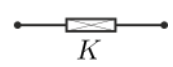

$(e)$

Figure 3

Process of constructing a lumped parameter $K$ corresponding to a three-dimensional tetrahedral element with material property $\kappa$

The previously described process can be represented formally as follows:

$K=\kappa \cdot \frac{\bar{A}}{R}=\kappa \cdot \frac{\frac{1}{R} \int_{T_{e}} A(r) d r}{R}=\kappa \cdot \frac{V}{R} \cdot \frac{1}{R}=\kappa \cdot \frac{V}{R^{2}}$

where $\mathrm{R}$ is the radius of the circumsphere. In the two-dimensional case, the process is similar to the three-dimensional case as shown in Fig. 4, but we start with a triangular element $T_{e}$ for this approach with a height $h$. The formula corresponding to the two-dimensional case is as follows:

$K=\kappa \cdot \frac{\bar{m} \cdot h}{R}=\kappa \cdot \frac{\frac{1}{R} \int_{T_{e}} m(r) d r}{R} \cdot h=\kappa \cdot \frac{A}{R} \cdot \frac{1}{R} \cdot h=\kappa \cdot \frac{A}{R^{2}} \cdot h=\kappa \cdot \frac{V}{R^{2}}$

where $\mathrm{R}$ is now the radius of the circumcircle. The calculation of the lumped parameter values must be performed for each sub-element in both the threedimensional (3) and the two-dimensional (2) cases.

\subsection{Graph Representation}

The main advantage of constructing a graph from a continuum compared to a traditional discretisation method (like FEM, FDM) is that since the graph is independent of the underlying physical space, the abrupt changes of material properties and boundary structures are handled in a straightforward manner, regardless of the dimension of the underlying space. Moreover, all the relevant 
results from discrete calculus and graph theory can be applied. Fig. 5 shows a few possible surface geometries represented by the same graph after the topological transformation. The original domain shapes are rectangular (a), semi-cylindrical (b), disk (c), and hemispherical (d) respectively. Regardless of the shape of the original geometry, the matrices that represent the topology of the graph will be the same. The only difference is in the actual weights of the graph edges.

The definition and most important properties of the graph used are the followings

$G=(V, E)$

where $V$ is the set of vertices and $E$ is the set of edges, with weights $w: E \rightarrow C$. The real and imaginary parts of the weights are physical properties corresponding to the material in the physical domain. The discretisation operator is defined as:

$D: \Omega \times K \rightarrow G$

where $\Omega \subset \mathrm{R}^{n}$ is the physical domain, $n$ is the number of space-dimensions, $K$ is the set of physical parameters, and $G$ is the set of graphs with properties defined in (4). The operator defined in (5) can be applied to an ample set of physical problems (processes which can be represented by elliptic PDEs), and the discretisation always leads to a linear system of equations corresponding to a lumped parameter model. 


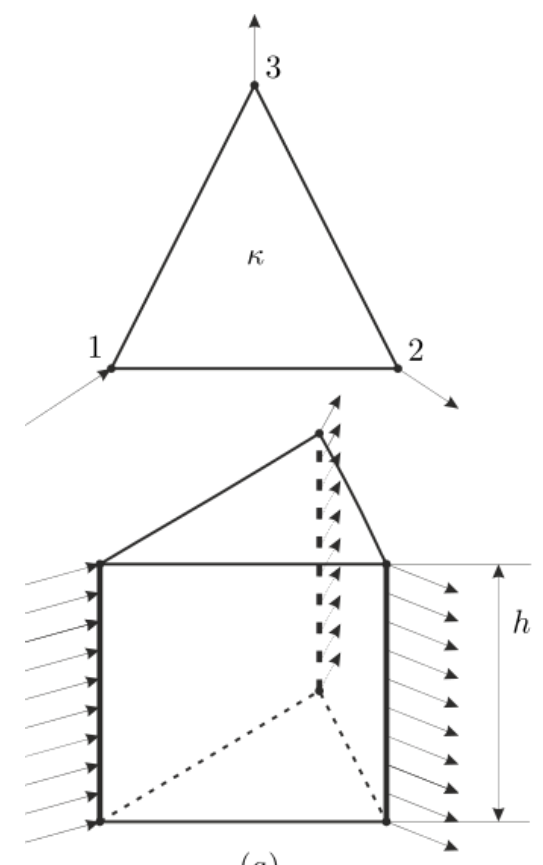

(a)

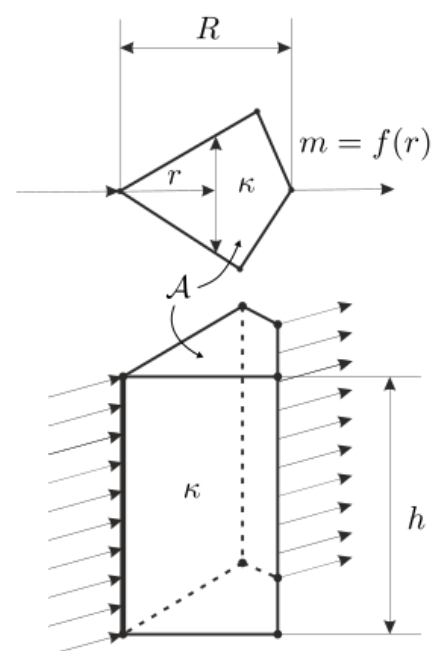

(c)

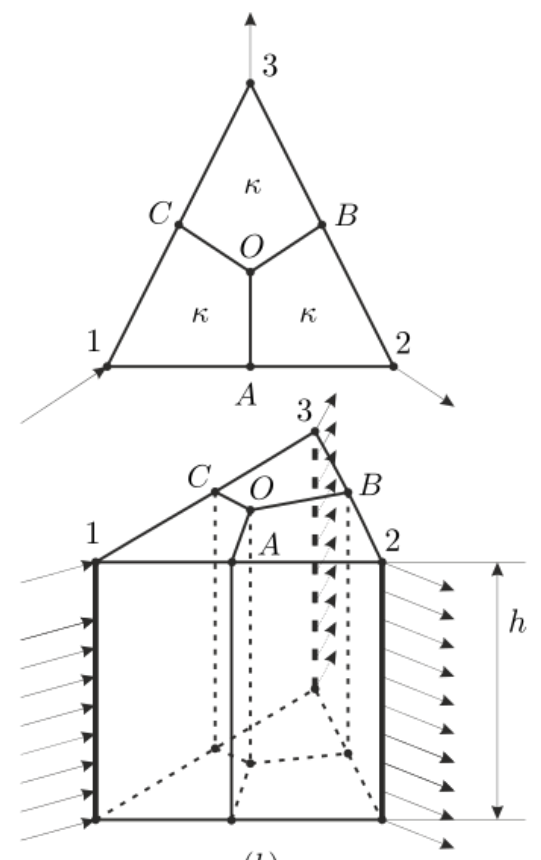

(b)
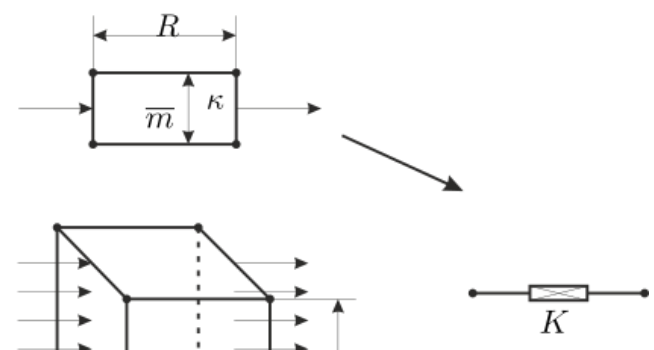

(e)

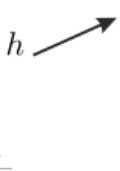

$(d)$

Figure 4

Process of constructing a lumped parameter $K$ corresponding to a two-dimensional triangular element with material property $\kappa$ 


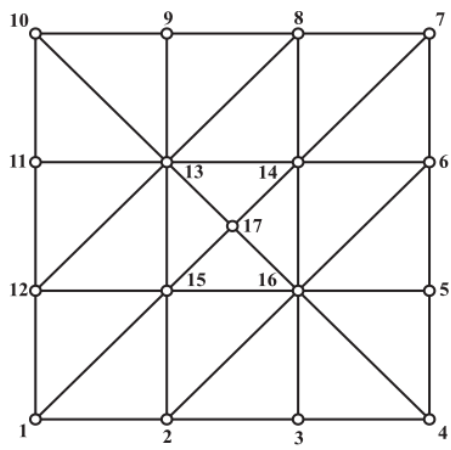

(a)

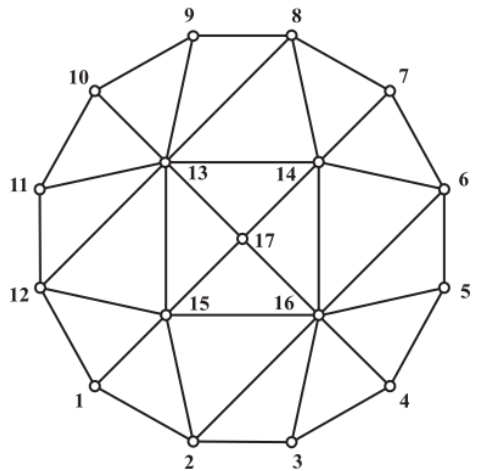

(c)

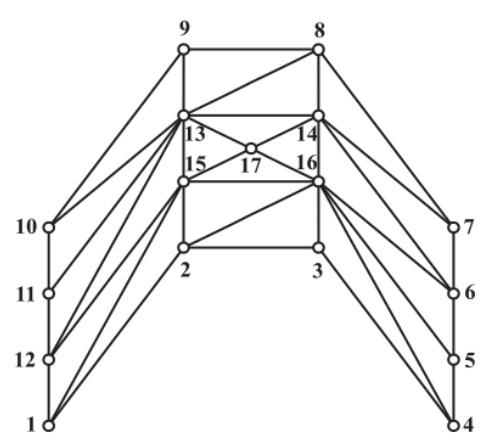

(b)

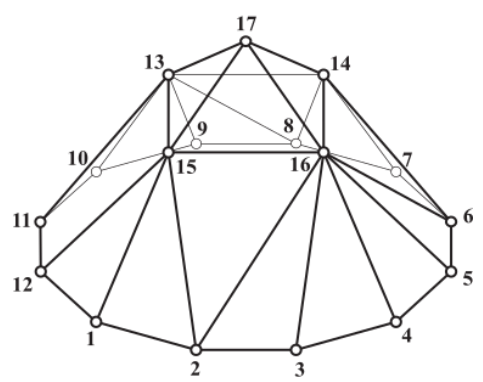

(d)

Figure 5

Different surface geometries represented by the same graph

Table 1 represents a comparison between the continuous and discrete versions of a few important differential operators.

Table 1

Analogy between continuous and discrete operators [25]

\begin{tabular}{c|c|c} 
Name & Continuous & Discrete \\
\hline Kirchhoff Current Law & $\nabla \cdot J=s$ & $\mathbf{A} \cdot \mathbf{j}_{\mathbf{b}}=\mathbf{j}_{\mathbf{s}}$ \\
\hline Kirchhoff Voltage Law & $\nabla \times \nabla u=0$ & $\mathbf{B}^{\mathbf{T}} \cdot \mathbf{u}_{\mathbf{b}}=\mathbf{0}$ \\
\hline Linear transport equation & $J=-\kappa \cdot \nabla u$ & $\mathbf{j}_{\mathbf{b}}=-\mathbf{K} \cdot \mathbf{A}^{\mathbf{T}} \mathbf{u}_{\mathbf{n}}$ \\
\hline Elliptic transport equation & $-\nabla \cdot(\kappa \cdot \nabla u)=s$ & $-\left(\mathbf{A K A} \mathbf{A}^{\mathbf{T}} \cdot \mathbf{u}_{\mathbf{n}}\right)=\mathbf{j}_{\mathbf{s}}$
\end{tabular}

In Table $1 \mathbf{J}$ is the extensive current density, $\mathbf{A}$ is the node-branch incidence matrix, $\mathbf{B}$ is the branch-loop incidence matrix, $\mathbf{K}$ is a diagonal matrix of weights on the edges of the graph, $\mathbf{j}_{\mathbf{b}}$ is the extensive current on the branches, $\mathbf{j}_{\mathbf{s}}$ is the extensive source current, $\mathbf{u}_{\mathbf{n}}$ is the potential in the nodes, and $\mathbf{u}_{\mathbf{b}}=\mathbf{A}^{\mathbf{T}} \cdot \mathbf{u}_{\mathbf{n}}$ is the 
potential difference (intensive quantity) on the branches. All of the discrete operators enlisted in Table 1 have the same advantageous properties (orthogonality, conservation, minimum-maximum principle) as those of their continuous counterparts [25]. Due to the strict approach, our proposed method guarantees that the resulting discrete operator always leads to a solvable system of linear equations (for example in the case of elliptic transport, the resulting discrete operator will be a Laplacian matrix interpreted on a graph).

To determine the solution of a PDE, we generally need a boundary condition. In the case of solving the discrete version of the problem on a graph (which is loosely connected to the geometry of the physical domain), we need to extend the graph using virtual branches (Fig. 6). This set of branches form the boundary, and all generally used boundary conditions (Dirichlet, Neumann, Outward/Inward Flux, etc.) can be defined on these branches.

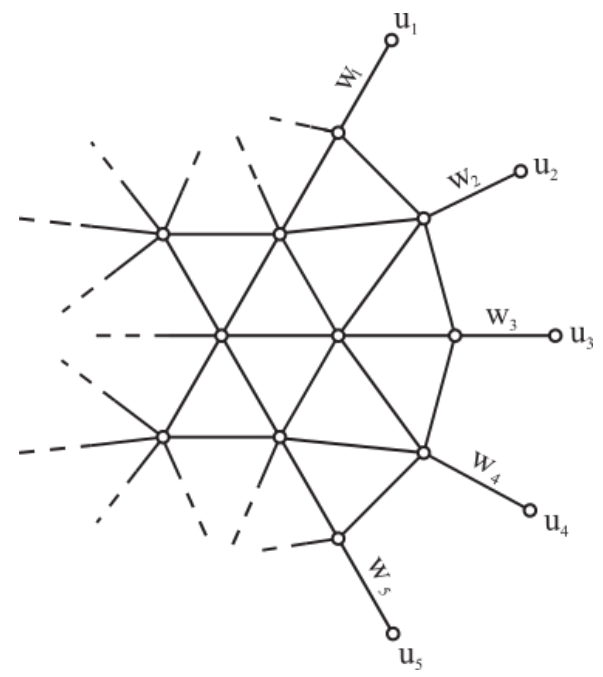

Figure 6

Boundary conditions represented by virtual branches

Currently, we have investigated the Neumann and Dirichlet boundary conditions only since these are the most frequently occuring ones. Even in case of heatflow problems, we can handle certain boundaries as Dirichlet types while others as Neumann type boundaries, but not the mixed version in our current implementation. As a future work, the inclusion of a third type boundary condition is also planned to make our method more flexible. During the definition of boundary conditions, the values of the weights $w_{i}$ can be zero, or infinity as well. For example, a particular Dirichlet boundary condition can be handled by a virtual edge with $w_{i} \rightarrow \infty$ rendering the potential of the inside node equal to the prescribed potential of the outside node (i.e. ground node, $u_{i}=0$ in the electric case). In the case of the graph representation, the handling of the sources can be 
performed by adding potential and current sources for any of the nodes of the graph (see Table 2).

Table 2

Fundamental boundary conditions defined by virtual branchesImplementation of the method

\begin{tabular}{c|c|c|c} 
Boundary condition & Weight & Potential & Flux \\
\hline Dirichlet & $w$ & $u$ & - \\
Neumann & $w$ & - & $i$ \\
Insulation & 0 & - & -
\end{tabular}

Concerning the special approach and the properties of the proposed method, we decided to describe the technique as the Lumped Element Method (LumEM). As an introductory model example, let us assume we have a sufficiently small domain of a 2D space, where the material properties can be assumed to be homogeneous. In the case of such a simple domain, the procedure of discretisation is given in the following section.

As the first step of discretisation the domain has to be partitioned into a triangular mesh (Delaunay triangulation), where the definitions are as follows:

- $\quad M:$ mesh

- $\quad M^{0}:$ nodes (vertices, points)

- $\quad M^{1}$ : branches (edges, lines)

- $\quad M^{2}$ : triangles (faces, loops)

In the following subsections, we examine the steps of discretisation for two simple geometries containing one and two triangles.

\subsection{Simple Disk with One Triangle}

In the case of a simple disk domain assuming homogeneous material properties $\kappa$ containing one triangle, Fig. 7 shows the process of transforming the material property $\kappa$ of the homogeneous material to the edges of a triangle. The methods shown below can be used during discretization for any triangle created by Delunay triangulization. The boundary of the domain has three nodes where extensive flux can flow into or out of the domain. The triangular domain is subdivided into three smaller domains (see $A_{1}, A_{2}, A_{3}$ in Fig. 7) by connecting the centre of the circumcircle to the midpoints of the sides. 

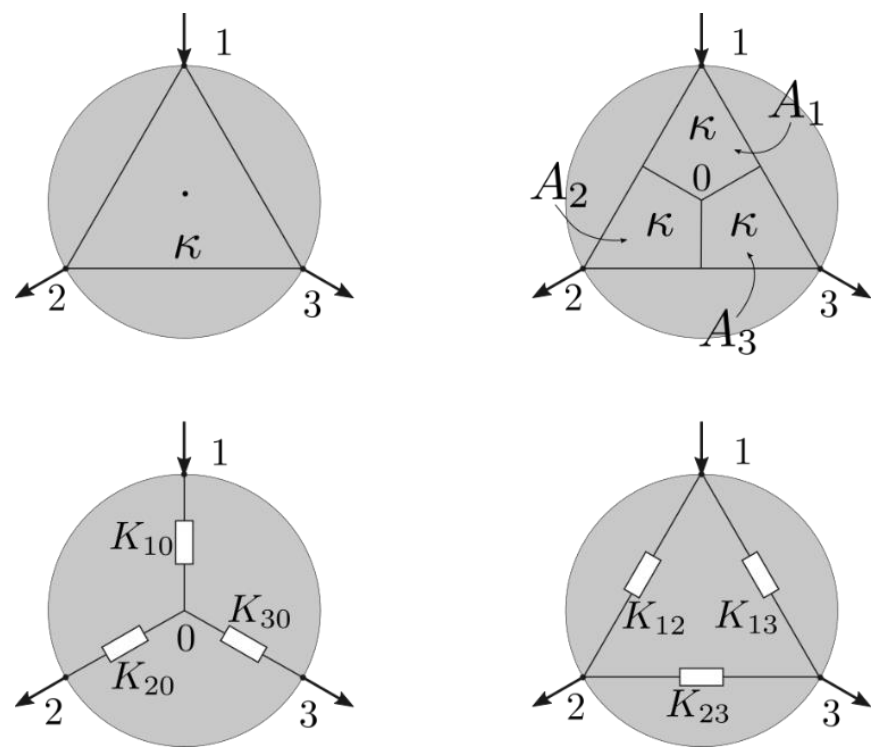

Figure 7

Development of a transport coefficient network for one triangle

After dividing the domain into three parts, each area can be replaced by an edge with a weight that concentrates the $\kappa$ of the domain to the edge. The edges are constructed by connecting the vertices of the triangle to the centre of the circumcircle, hence the lengths of the resulting edges are equal. Mapping the material property $\kappa$ to the edges can be done by the following set of rules

$K_{i, 0}=\kappa \frac{A_{i}}{R^{2}} h, \quad i=1,2,3$

where the resulting $K_{(i, 0)}$ can be interpreted as the concentrated material properties which will form graph edges after a star-delta conversion as follows:

$K_{i, j}=K_{j, i}=\frac{K_{i, 0} K_{j, 0}}{\sum_{i=1}^{n} K_{i, 0}}=\kappa \frac{h}{R^{2}} \frac{A_{i} A_{j}}{\sum_{\ell=1}^{n} A_{\ell}}=\kappa t_{i, j}, \quad(i, j=1,2,3, i \neq j)$

The weights $K_{(i, j)}$ on the resulting edges are equal because of the assumption of a homogeneous distribution of material properties on the disk. As such, the disk domain is transformed into a triangular subgraph.

\subsection{Simple Disk with Two Triangles}

In the next case, the disk-shaped domain is decomposed into two disjunct triangles (Fig. 8). The boundary of the domain has four nodes where extensive flux can flow into or out of the domain. The transport coefficients $\kappa_{1}$ and $\kappa_{2}$ on the triangular domains are assumed to be homogeneous inside each triangle. From this 
point onwards, the triangles are treated in a similar way to the case of the onetriangle example in the preceding section as seen in (8) and (9):

$K_{i, P}=\kappa^{(1)} \frac{A_{i}^{(1)}}{\left(R^{(1)}\right)^{2}} h, \quad(i=1,2,3)$

$K_{i, Q}=\kappa^{(2)} \frac{A_{i}^{(2)}}{\left(R^{(2)}\right)^{2}} h, \quad(i=1,2,3)$

where $R^{(1)}, R^{(2)}$ are the radii of the circumcircles of triangles 1 and 2 respectively.

After this, we perform a Y- $\Delta$ transformation on each triangle, described by the following formulas:

$K_{i, j}^{(1)}=K_{j, i}^{(1)}=\kappa^{(1)} t_{i, j}^{(1)},(i, j=1,2,3, i \neq j)$

$K_{i, j}^{(2)}=K_{j, i}^{(2)}=\kappa^{(2)} t_{i, j}^{(2)},(i, j=1,2,3, i \neq j)$

where $t_{i, j}^{(1)}, t_{i, j}^{(2)}$ are the factors corresponding to the subdomains 1 and 2 respectively, with the same form as introduced in (7). As a result of the Y- $\Delta$ transformation, there will be a pair of edges $\left(K_{1,3}^{(1)}, K_{1,3}^{(2)}\right)$ which connect the same two nodes. In this case, the resulting transport coefficient is the sum of those of the branches according to the characteristics of the flow of the extensive flux.
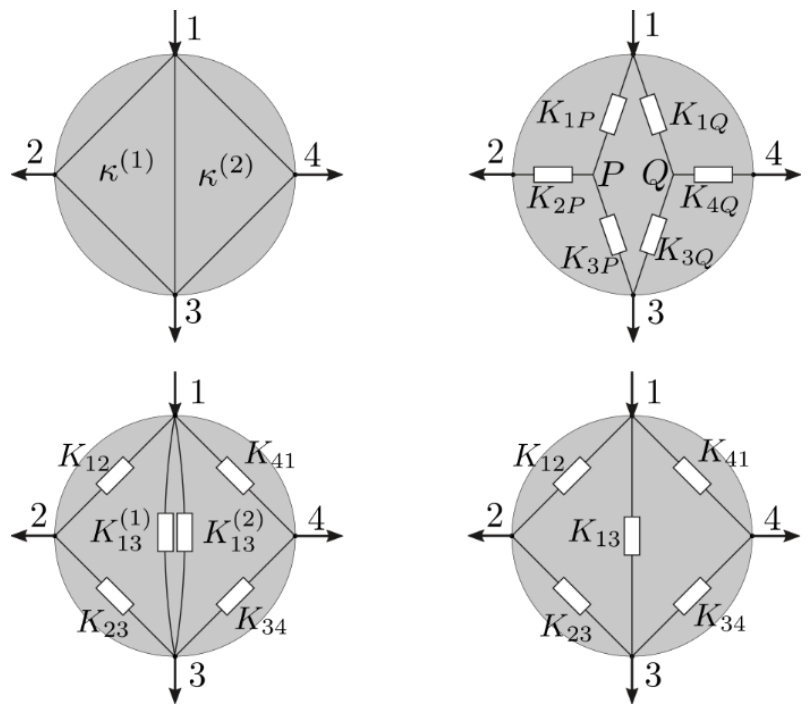

Figure 8

Development of a transport coefficient network for two adjacent triangles 
Based on this outlined concept, the weights of the resulted graph are as follows:

$$
\left\{\begin{array}{l}
K_{1,2}=\kappa^{(1)} t_{1,2}^{(1)} \\
K_{2,3}=\kappa^{(1)} t_{2,3}^{(1)} \\
K_{3,4}=\kappa^{(2)} t_{3,4}^{(2)} \\
K_{4,1}=\kappa^{(2)} t_{4,1}^{(2)} \\
K_{1,3}=\kappa^{(1)} t_{1,3}^{(1)}+\kappa^{(2)} t_{1,3}^{(2)}
\end{array}\right.
$$

which can be written in matrix form as:

$\left[\begin{array}{cc}t_{1,2}^{(1)} & 0 \\ t_{2,3}^{(1)} & 0 \\ 0 & t_{3,4}^{(2)} \\ 0 & t_{4,1}^{(2)} \\ t_{1,3}^{(1)} & t_{1,3}^{(2)}\end{array}\right]\left[\begin{array}{l}\kappa^{(1)} \\ \kappa^{(2)}\end{array}\right]=\left[\begin{array}{l}k_{1,2} \\ k_{2,3} \\ k_{3,4} \\ k_{4,1} \\ k_{1,3}\end{array}\right]$

from this, a compact matrix form can be

$$
\mathbf{T} \cdot \mathbf{K}=\mathbf{k}
$$

where $\kappa$ is the vector of transport coefficients corresponding to triangular domains, $\mathbf{k}$ is the vector of weights of the resulting graph, and $\mathbf{T}$ is a transformation matrix. Equations (12) and (13) illustrate the essence of the material discretisation process described in Section 2, according to which the discontinuity of material properties taken as constant on the elements are smoothed at the connections of the elements.

The transformation matrix $\mathbf{T} \in \mathrm{R}^{\left|M^{1}\right| \times\left|M^{2}\right|}$ has only three non-zero values $t_{j}$ in the $\mathrm{j}$-th column, corresponding to the incident branches of the $\mathrm{j}$-th triangle ${ }^{1}$. The representation of the boundary branches in the matrix ensures full column rank for any $\mathbf{T}$. Because of this property, there exists a pseudo-inverse $\mathbf{T}^{+}$of $\mathbf{T}$, and (14) can also be solved for $\kappa$ in view of $\mathbf{T}$ and $\mathbf{k}$. Thus, the subdivision procedure described can be easily generalised to an arbitrary number of triangles.

\section{Case Studies}

To demonstrate the effectiveness and robustness of the method, case studies have been created for which the results obtained by the LumEM method are illustrated and compared with a well-known FEM solving procedure realized in COMSOL

Matrix $\mathbf{T}$ is essentially a branch-loop incidence matrix. 
Multiphysics environment. In the case studies, we will discuss in detail the implementation of the LumEM method. During discretization, using the same mesh for both methods, the LumEM and FEM potential values assigned at the nodes become easy and comparable. In addition, relative errors between the two methods are calculated and graphically interpreted. In the first case study, we construct a fundamental electrical problem and then modify it to generate a numerical example that demonstrates the robustness of the LumEM method, even with unrealistic material property variation. In the third case, we solve a fundamental heat transfer problem using the LumEM method in order to show that the method is also consistent with time-dependent parabolic PDEs. Of course, a comparison with FEM is carried out for each case study.

\subsection{Basic Electric Problem}

In this section, an application of the previously described discretisation method is presented for a simple electric problem, assuming time-harmonic functions. Let's assume a circular domain (with a diameter of $1 \mathrm{~m}$ ) shown in Fig. 9 with given physical parameters.

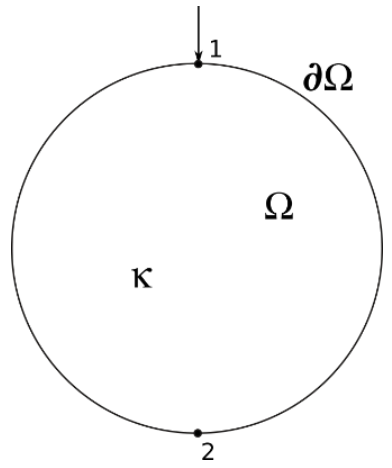

Figure 9

Simple domain

$\kappa_{i}=\sigma_{i}+j \omega \varepsilon_{i}$

where $\sigma_{i}$ is the conductivity, $\varepsilon_{i}$ is the relative permittivity, $i=1 \ldots M^{2}$. After discretisation, the parameters corresponding to the branches of the resulting graph, are given as:

$K_{k}=G_{k}+j \omega C_{k}$

where $G_{k}$ is the conductance, $C_{k}$ is the capacitance, $k=1 \ldots M^{1}$. The fundamental equation describing the problem examined is given as:

$\nabla \cdot(\kappa \nabla u)=0$ 
Since $\kappa$ is a constant over the whole domain $\Omega$, the following form can be written:

$\Delta u=0$

The mathematical model can be used to mimic a physical problem in which a voltage generator is connected to a single point $\left(N_{1}\right)$ on the boundary. The ground point is also placed at a highlighted point on the boundary $\left(N_{2}\right)$. The remaining part of the boundary $(\Gamma)$ is formed from electrical insulating material. Another purpose of this case study is to demonstrate that the method can be easily and efficiently applied to point sources.

To define the boundary conditions, we denote the potentials on the boundary by $\phi(s)$, where the argument $s$ is the normalised boundary length $(s \in[0,1])$. The applied boundary conditions are a Neumann-boundary $i_{g} \delta(s=0)$ at node $N_{1}$ and a Dirichlet-boundary $\phi(s=1 / 2)=\phi_{0}=0$ (ground) at node $N_{2}$. For all of the other boundary nodes (in $\Gamma$ ), the boundary condition is given as:

$\mathbf{n} \cdot \nabla \phi(s)=0 \quad s \in \Gamma$

where $\partial \Omega=\Gamma+N_{1}+N_{2}$.

The solution steps are as follows:

1. Construct a triangulation of the investigated domain (for example using the Distmesh algorithm [26]).

2. Transform the domain into a graph by applying (14).

3. Identify solution of the equation system

$\mathbf{A K A}^{\mathbf{T}} \mathbf{u}=\mathbf{i}_{\mathbf{g}}$

where $\mathbf{A} \in\{0,1,-1\}^{\left|M^{0}\right| \times\left|M^{1}\right|}$ is the reduced incidence matrix ${ }^{2}, \mathbf{K} \in \mathrm{C}^{\left|M^{1}\right| \times\left|M^{1}\right|}$ is a diagonal matrix built from the elements of $\mathbf{k}$ (see 3.2 Section), $\mathbf{u} \in \mathrm{C}^{\left|M^{0}\right|}$ is the vector of potentials, and $\mathbf{i}_{\mathrm{g}} \in \mathrm{C}^{\left|M^{0}\right|}$ is the vector of generator currents. The matrix $\mathbf{A K \mathbf { A } ^ { \mathbf { T } }}$ in (20) can be interpreted as a Laplace-operator (weighted Laplacian matrix) [25] for the nodes.

\subsection{Basic Parabolic Problem}

In this section, the discretisation method is applied to a basic time-dependent heat conduction problem. Similarly to the electric problem, we assume a circular domain (with a diameter of $1 \mathrm{~m}$ ) shown in Fig. 10, with given physical parameters

$\kappa_{i}=k_{i}$

2 The reduced incidence matrix is created by deleting the row corresponding to the ground node from the matrix $\mathbf{A}$ [70]. 
where $k_{i}$ is the thermal conductivity.

The fundamental equation defining the problem is

$\rho c_{p} \frac{\partial u}{\partial t}+\nabla \cdot(-\kappa \nabla u)=0$

where $u$ is the temperature, $\rho$ is the density, $c_{p}$ is the specific heat capacity, and $\kappa$ is the thermal conductivity. Since $\kappa$ is constant over the entire domain (22) can be written as:

$\rho c_{p} \frac{\partial u}{\partial t}-\kappa \Delta u=0$

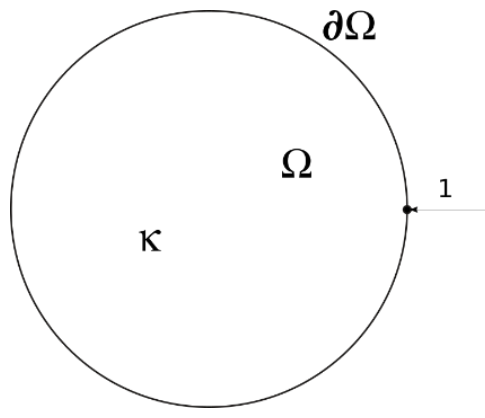

Figure 10

Simple domain for a parabolic problem

The boundary conditions applied are a Dirichlet-boundary $\phi_{0}$ at node $N_{1}$. For all of the other boundary nodes, the boundary condition is given as:

$\mathbf{n} \cdot \nabla \phi(s)=0 \quad s \in \Gamma$

where $\partial \Omega=\Gamma+N_{1}$. Of course, for non-homogeneous Dirichlet boundary conditions, the function is evaluated in the nodes on the boundary and these values are used to implement the solver.

The solution steps are as follows:

1. Construct a triangulation of the investigated domain (for example using the Distmesh algorithm [26]).

2. Transform the domain into a graph by applying (14).

3. Identify a solution of the following equation system by applying the backward-Euler method for discretisation of the time derivative.

$\rho c_{p} \frac{\mathbf{u}_{i+1}-\mathbf{u}_{i}}{\Delta t}-\mathbf{A K A}^{\mathrm{T}}\left(\mathbf{u}_{i+1}\right)=0$

denoting the relevant time steps by indexes $i$ and $i+1$. From this form, we can rearrange the terms to get: 
$-\frac{\rho c_{p}}{\Delta t} \mathbf{u}_{i}=\left(\mathbf{L}_{\mathbf{\kappa}}-\frac{\rho c_{p}}{\Delta t} \mathbf{I}\right) \mathbf{u}_{i+1}=\mathbf{M} \mathbf{u}_{i+1}$

where $\mathbf{L}_{\mathbf{K}}=\mathbf{A} \mathbf{K} \mathbf{A}^{\mathbf{T}}$ is the discrete Laplace operator (weighted Laplacian matrix) [70] containing the material property $\kappa$. Based on this result, with the introduction of the matrix $\mathbf{M}$, we have the following form:

$\mathbf{u}_{i+1}=-\frac{\rho c_{p}}{\Delta t} \mathbf{M}^{-1} \mathbf{u}_{i}$

The solution of (27) can be acquired by iterating over the time steps starting from an initial condition $u_{0}$ and taking into account the boundary conditions.

\section{Results and Discussion}

\subsection{Solution of the Electric Problem}

In the first example (in 4.1 Section) we have assumed a homogeneous material distribution over the whole domain, and the value of the transport coefficient $\kappa$ defined in (15) is $\kappa=1[S / m]+j \omega 1[F / m]$ (assuming a fixed $\omega=1[1 / s]$ ) and according to the two-dimensional nature of the problem, the domain is assumed to have unit height $h=1[\mathrm{~m}]$. In the formula we have substituted the numerical value into epsilon, the construction of epsilon based on relative permittivity is also applicable without any complications.

Solving (20) provides the potentials u. The solution can be seen in Fig. 11 compared to a solution calculated using the COMSOL FEM environment. The figure shows the nodal values of the potential and the relative error calculated as

$\epsilon=\frac{\left|u_{n}-u_{r e f}\right|}{\max \left(u_{r e f}\right)}$

It can be seen from the figure that there is a very good agreement (the maximum relative error is less than $3 \%$ ) between our solution and the reference solution (by COMSOL).

The next example covers a non-homogeneous material distribution, where there is a concentric disk $\left(x_{0}=0, y_{0}=0, r=0.35\right)$ inside the domain, where the transport coefficient is $\kappa_{1}=10^{10}+10^{10} j$, and the remaining area has a transport coefficient $\kappa=1+j$.

Fig. 12 shows a comparison of the calculated potential fields with the corresponding relative error. From the figure, it is clear that there is an excellent agreement for the non-homogeneous case as well since the maximum relative error is approximately $4 \%$. 


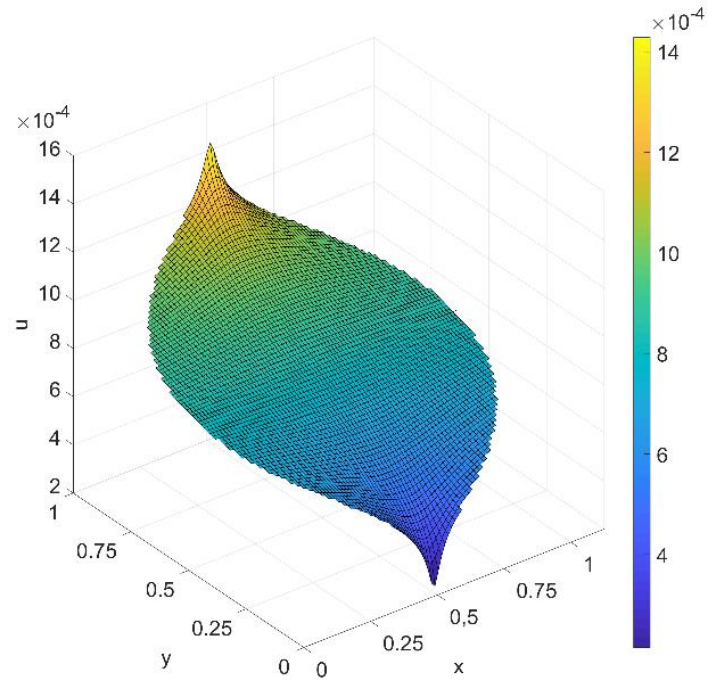

Figure 11

The potential distribution calculated by LumEM method (a), the comparison of numerically calculated potentials (b), and the corresponding relative errors (c) for the homogeneous case

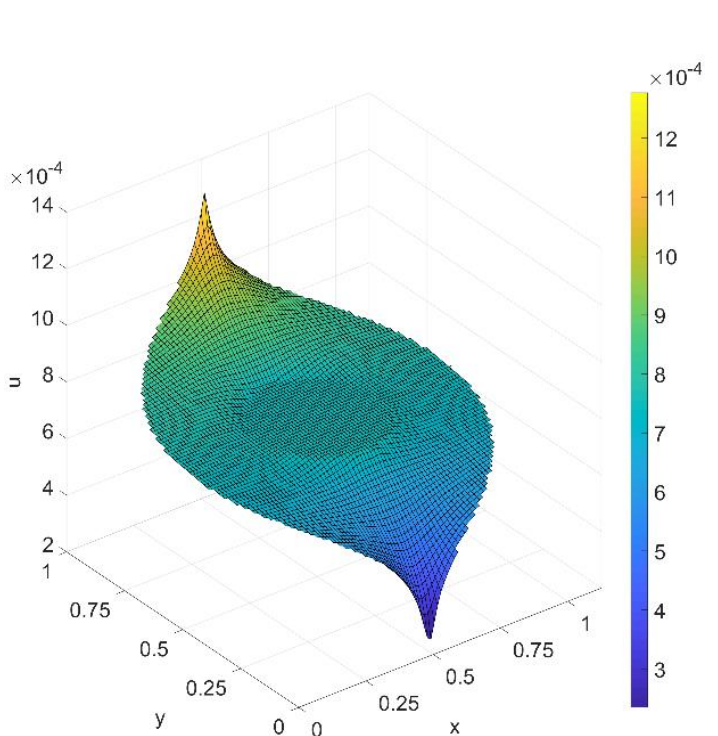

(a)

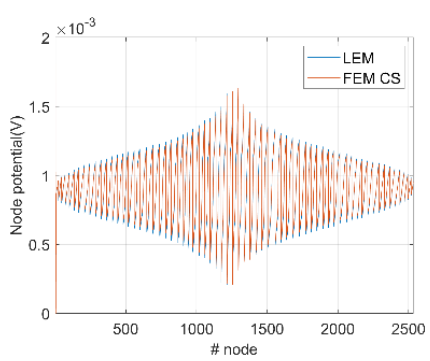

(b)

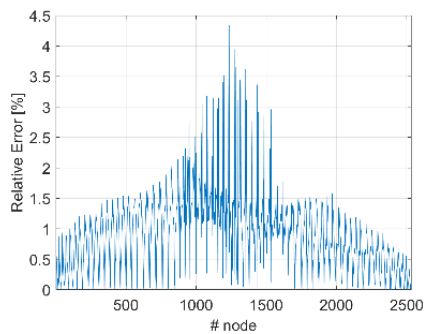

(c)

Figure 12

The potential distribution calculated by LumEM method (a), the comparison of numerically calculated potentials (b), and the corresponding relative errors (c) for the non-homogeneous case 


\subsection{Solution of the Heat Transfer Problem}

The actual parameters used for the heat transfer problem discussed in Section 4.2 are the thermal conductivity $k=1[W /(m K)]$, density $\rho=1\left[\mathrm{~kg} / \mathrm{m}^{3}\right]$, and specific heat capacity $c_{p}=1[\mathrm{~W} /(\mathrm{kg} \mathrm{K})]$. According to the two-dimensional nature of the problem, the domain is assumed to have a unit height $h=1[\mathrm{~m}]$.

During the solution of (27), we assumed a $1 \mathrm{sec}$ interval during time-stepping and we have investigated the solution at the last time step corresponding to $t=1 \mathrm{sec}$. Fig. 13 shows the solution compared to those obtained from COMSOL and the corresponding relative errors taking the COMSOL solution as the basis of the comparison. The average relative error is $1.32 \%$. The maximal values of the errors located in the close vicinity of the boundary node (large node indexes), where the temperature changes very sharply according to a Dirac-like boundary condition.

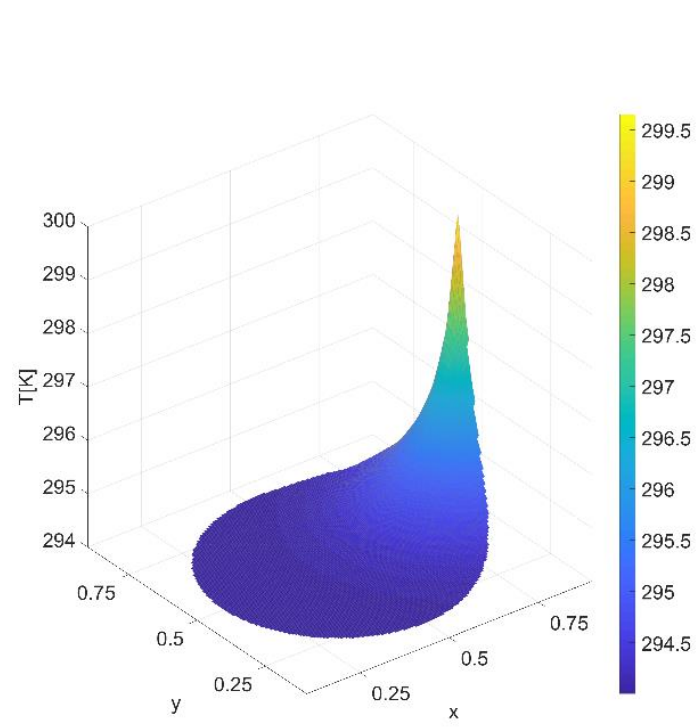

(a)

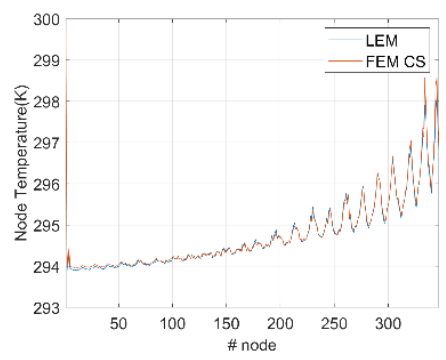

(b)

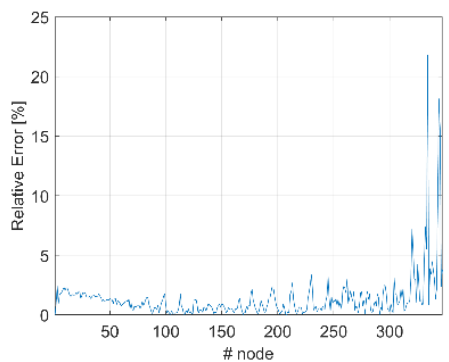

(c)

Figure 13

The temperature distribution calculated by LumEM method (a), the comparison of numerically calculated temperature values (b), and the corresponding relative errors (c) for the heat transfer problem

\section{Conclusion and Future Work}

In this report, we have introduced a new discrete calculus-based approach for the solution of elliptic and parabolic type linear partial differential equations. Based on the special properties of the proposed method, it was called the Lumped Element Method. This is because the approach relies on a special topological 
transformation from the continuous domain to a weighted graph containing lumped parameters that correspond to the material properties. We implemented the approach in the solution of two different model problems and investigated the results of the numerical solutions. It was determined that the proposed method is applicable to obtain the numerical solution of linear elliptic and parabolic PDEs. A future research goal is the generalisation of the method to a broader class of PDEs (hyperbolic, non-linear, etc.) and the investigation of the possible applications of the method to more complicated topologies and 3D problems. In addition, the method provides an opportunity to extend it towards analytical solving methods with one dimension or even a higher dimension [27].

\section{Acknowledgement}

This research was funded by project EFOP-3.6.1-16-2016-00004 and 2020-4.1.1TKP2020, co-financed by the Ministry for Innovation and Technology in Hungary and the European Union.

\section{References}

[1] Hatami, M., (2017) Weighted Residual Methods. Elsevier Science Publishing Co Inc. [30] Hiptmair, R., 2001, Discrete hodge-operators: An algebraic perspective. Progress In Electromagnetics Research 32, 247-269

[2] Mazumder, S., (2015) Numerical Methods for Partial Differential Equations. Elsevier Science Publishing Co Inc.

[3] Majumder, P., Eldho, T. I., (Feb 2016) A new groundwater management model by coupling analytic element method and reverse particle tracking with cat swarm optimization. Water Resources Management 30 (6), 19531972

[4] Asaithambi, R., Mahesh, K., (Jul 2017) A note on a conservative finite volume approach to address numerical stiffness in polar meshes. Journal of Computational Physics 341, 377-385

[5] Shi, C., Shu, C.-W., (Jun 2018) On local conservation of numerical methods for conservation laws. Computers \& Fluids 169, 3-9

[6] Petras, A., Ling, L., Ruuth, S., (Oct 2018) An RBF-FD closest point method for solving PDEs on surfaces. Journal of Computational Physics $370,43-57$

[7] Serkh, K., Rokhlin, V., (Jan 2016) On the solution of elliptic partial differential equations on regions with corners. Journal of Computational Physics 305, 150-171

[8] Coco, A., Russo, G., (May 2018) Second order finite-difference ghost-point multigrid methods for elliptic problems with discontinuous coefficients on an arbitrary interface. Journal of Computational Physics 361, 299-330 
[9] Hou, T. Y., Hwang, F.-N., Liu, P., Yao, C.-C., (May 2017) An iteratively adaptive multi-scale finite element method for elliptic PDEs with rough coefficients. Journal of Computational Physics 336, 375-400

[10] Toth, F., Kaltenbacher, M., (Oct 2016) Time and frequency domain finite element implementation of a fully anisotropic linear visco-elastic model. PAMM 16 (1), 397-398

[11] Lipnikov, K., Manzini, G., Shashkov, M., (Jan 2014) Mimetic finite difference method. Journal of Computational Physics 257, 1163-1227

[12] Frank, J., Moore, B. E., Reich, S., (Jan 2006) Linear PDEs and numerical methods that preserve a multisymplectic conservation law. SIAM Journal on Scientific Computing 28 (1), 260-277

[13] Nicolaides, R. A., Wu, X., (Dec 1997) Covolume solutions of threedimensional div-curl equations. SIAM Journal on Numerical Analysis 34 (6), 2195-2203

[14] Nicolaides, R., (Jun 1989) Flow discretization by complementary volume techniques. In: $9^{\text {th }}$ Computational Fluid Dynamics Conference. American Institute of Aeronautics and Astronautics

[15] Perot, B., (Mar 2000) Conservation properties of unstructured staggered mesh schemes. Journal of Computational Physics 159 (1), 58-89

[16] Perot, B., Nallapati, R., (Jan 2003) A moving unstructured staggered mesh method for the simulation of incompressible free-surface flows. Journal of Computational Physics 184 (1), 192-214

[17] Perot, J., (Sep 1993) An analysis of the fractional step method. Journal of Computational Physics 108 (1), 51-58

[18] Perot, J. B., (Oct. 1995) Comments on the Fractional Step Method. Journal of Computational Physics 121, 190-191

[19] Perot, J., Subramanian, V., (May 2007) Discrete calculus methods for diffusion. Journal of Computational Physics 224 (1), 59-81

[20] Perot, J. B., Vidovic, D.,Wesseling, P., (2006) Mimetic reconstruction of vectors. In: Compatible Spatial Discretizations. Springer New York, pp. $173-188$

[21] Chang, W., Giraldo, F., Perot, B., (Jul 2002) Analysis of an exact fractional step method. Journal of Computational Physics 180 (1), 183-199

[22] Desbrun, M., Hirani, A. N., Leok, M., Marsden, J. E., (2005) Discrete exterior calculus. arXiv:Math/0508341

[23] Wenneker, I., Segal, A., Wesseling, P., (Jan 2003) Conservation properties of a new unstructured staggered scheme. Computers \& Fluids 32 (1), 139147 
[24] Subramanian, V., (2007) Discrete calculus methods and their implementation. PhD. thesis, Mechanical Engineering, University of Massachusetts

[25] Leo J. Grady, J. P., (2010) Discrete Calculus. Springer London

[26] Persson, P.-O., Strang, G., (Jan 2004) A simple mesh generator in MATLAB. SIAM Review 46 (2), 329-345

[27] Vizvari, Z., Sari, Z., Klincsik, M. Odry, P. (2020) Exact schemes for second-order linear differential equations in self-adjoint cases. Adv Differ Equ, 497, https://doi.org/10.1186/s13662-020-02957-7 